\title{
Transmission Line Sequence Impedances Identification Using PMU Measurements
}

\author{
Huafeng Zhou ${ }^{1}$, Xuanyu Zhao ${ }^{1}$, Di Shi ${ }^{2}$, Huashi Zhao ${ }^{1}$ and Chaoyang Jing ${ }^{2}$ \\ 1. Grid Control Center, China Southern Power Grid, Guangzhou 510000, China \\ 2. Energy Management via Information Technology, Pasadena, CA 91107, USA
}

Received: December 04, 2014 / Accepted: December 15, 2014 / Published: February 28, 2015.

\begin{abstract}
An online TL (transmission line) impedance TPIS (transmission line parameter identification system) using PMU (phasor measurement unit) was recently developed and implemented at CSG (china southern power grid company). Traditional approaches for TL impedance calculation only approximate the effect of conductor sags and ignore the dependence of impedances on temperature variation. Utilizing PMU measurements may improve the accuracy of TL parameters calculation. The challenge is that the parameters identified are very sensitive to noise and errors in PMU measurements, which are difficult to quantify and can be uncertain under different system operating/loading condition. TPIS provides an innovative yet practical problem formulation for TL sequence parameter estimation based on least-squares with linear constraints. A bootstrapping-based resampling technique is developed and a new metric is proposed to determine the credibility of the estimated sequence impedances. This paper discusses the proposed methodologies, challenges, as well as implementation issues identified during the development of TPIS.
\end{abstract}

Key words: Sequence impedance, transmission line, PMU, constrained optimization.

\section{Introduction}

Accurate knowledge of TL (transmission line) impedance parameters is of vital importance in power system operations and planning, such as state estimation, transient stability, and is used as the basis for protective relay settings. Traditionally, TL parameters have been estimated based on the tower geometries, conductor dimensions, estimates of actual line length, conductor sag, and other factors [1]. These calculated parameters are based on assumptions and approximations. With the development of the PMU (phasor measurement unit)technology, synchronized phases offer the possibility of allowing accurate estimation of transmission line impedance parameters. CSG (China Southern Power Grid Company) recently developed and implemented an online PMU-based TPIS (transmission line parameter identification

Corresponding author: Chaoyang Jing, Ph.D., chief engineer, research fields: smart grids, power system dynamics, stability and control, PMU related applications. E-mail: c.jing@myemit.com. system).

CSG operates one of the world's most complex systems. CSG has the world's first $\pm 800 \mathrm{kV} \mathrm{DC}$ transmission project. Hybrid operation of AC and DC, west-east corridor with long-distance and huge-capacity, complicated network structure with renewable energy integration, striking problem on grid stability, and great challenge on operation control all are the features of the CSG's grid. Towards the development of smart grid and with increasing renewable integration, CSG has put tremendous efforts to maintain grid reliability, minimize costs for customers, and to create an open infrastructure that can take advantage of evolving tools and grid devices. A critical portion of these efforts are to set up and maintain an accurate model of the system. With a large number of PMUs widely installed in its system, CSG plans to validate, improve, and update the network parameters in its EMS database using the newly developed TPIS. 
Extensive research has been conducted in identifying TL impedances using synchrophasor measurements [1-6] Four methods based on TL linear/nonlinear models were presented and compared in Ref. [1]. One two-port ABCD parameter based method is proposed in Ref. [2]. This method utilizes two samples of PMU measurements from each terminal of the TL to identify the ABCD parameters, from which the impedance parameters can be calculated. Ref. [3] proposes a method based on the distributed TL model and uses nonlinear estimation theory to generate an optimal estimator of the fault location as well as TL parameters. Ref. [4] proposed to use the full TL model for parameter identification if the line is not fully transposed and system is unbalanced. An extended Kalman filter based approach was proposed in Ref. [5]. As pointed out by Shi [6], PMU-based TL parameter identification is very demanding on the quality of the measurements. In other word, small errors in the phase measurements can lead to huge errors in the estimated impedance parameters. Recently, people started to pay more attention to PMU data quality issues and lots of efforts have been spent in developing methods for PMU calibration [7-9]. Generally speaking, the biggest challenge in this field still lies in the fact that TL parameter identification requires highly accurate PMU data while the accuracy of PMU measurements under different working/system conditions can be uncertain.

This paper proposes a new method for calculating TL sequence impedances using constrained optimization considering various physical constraints. It is recognized that existing methods neglect to include physical constraints into the parameter identification process. During the development of TPIS, it is realized that including physical constraints can be very helpful based on which a novel yet practical problem formulation and solution are proposed for such problems. In addition, a new metric is proposed which can be used to determine the credibility of the calculated parameters, which is missing in the literature.
This paper discusses the methodologies, challenges, as well as several implementation issues noticed during the development of TPIS.

\section{Problem Formulation and Solution}

\subsection{Measurement Model}

A three-phase equivalent model for a general transmission line is shown in Fig.1, where $\bar{V}_{a b c}^{S}, \bar{V}_{a b c}^{R}$, $\bar{I}_{a b c}^{S}, \bar{I}_{a b c}^{R}$ represent the three-phase voltage and current phasor vectors at both ends of the line while $Z_{a b c}$ and $Y_{a b c}$ are the series impedance matrix and shunt admittance matrix. Matrix $B_{a b c}$ is the three-phase shunt susceptance matrix of the line. Based on nodal analysis, the following equations can be written as:

$$
\begin{gathered}
\bar{V}_{a b c}^{S}-\bar{V}_{a b c}^{R}=Z_{a b c} \bar{I}_{a b c}^{S}-\frac{j}{2} \cdot Z_{a b c} \cdot B_{a b c} \cdot \bar{V}_{a b c}^{S} \\
\bar{I}_{a b c}^{S}+\bar{I}_{a b c}^{R}=\frac{j \cdot B_{a b c}}{2} \cdot\left(\bar{V}_{a b c}^{S}+\bar{V}_{a b c}^{R}\right)
\end{gathered}
$$

Voltage and current variables in the equations are all phase-frame-of-reference quantities. In order to transform these quantities to sequence-frame-of-reference quantities, it is necessary to apply the phase-to-sequence transformation matrix, which is defined as:

$$
A=\left[\begin{array}{ccc}
1 & 1 & 1 \\
1 & a^{2} & a \\
1 & a & a^{2}
\end{array}\right] \text { and } a=e^{j 120^{0}}
$$

The following relationships hold between the phase quantities and sequence quantities:

$$
\begin{gathered}
\bar{V}_{a b c}^{S(R)}=A \cdot \bar{V}_{012}^{S(R)} \\
\bar{I}_{a b c}^{S(R)}=A \cdot \bar{I}_{012}^{S(R)} \\
Z_{012}=A^{-1} Z_{a b c} A \\
B_{012}=A^{-1} B_{a b c} A
\end{gathered}
$$

where, $\bar{V}_{a b c}^{S(R)}$ and $\bar{I}_{a b c}^{S(R)}$ are the three-phase voltage and current measurement vectors for the sending end or the receiving end. $\bar{V}_{012}^{S(R)}$ and $\bar{I}_{012}^{S(R)}$ are the sequence voltage and current vectors for the sending end or the 


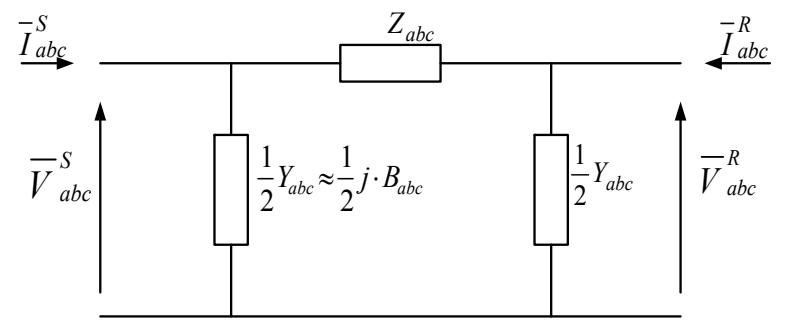

Fig. 1 Transmission line PI model.

receiving end. $Z_{012}$ is the sequence series impedance matrix of the transmission line.

In order to simplify Eq. (1), matrix $G_{a b c}$ is defined as:

$$
G_{a b c}=Z_{a b c} \cdot B_{a b c}
$$

So that Eq. (1) can be rewritten as:

$$
\bar{V}_{a b c}^{S}-\bar{V}_{a b c}^{R}=Z_{a b c} \cdot \bar{I}_{a b c}^{S}-\frac{j}{2} \cdot G_{a b c} \cdot \bar{V}_{a b c}^{S}
$$

For a transmission line, both the impedance matrix $Z_{a b c}$ and susceptance matrix $B_{a b c}$ are symmetrical. Therefore, matrix of $G_{a b c}$ is also symmetrical, as shown below:

$$
G_{a b c}=\left[\begin{array}{ccc}
G_{a} & G_{a b} & G_{a c} \\
G_{a b} & G_{b} & G_{b c} \\
G_{a c} & G_{b c} & G_{c}
\end{array}\right]
$$

Rewriting Eqs. (2) and (9) into matrix form:

$$
\begin{aligned}
& {\left[\begin{array}{c}
\Delta V_{a} \\
\Delta V_{b} \\
\Delta V_{c}
\end{array}\right]=\left[\begin{array}{ccc}
Z_{a} & Z_{a b} & Z_{a c} \\
Z_{a b} & Z_{b} & Z_{b c} \\
Z_{a c} & Z_{b c} & Z_{c}
\end{array}\right]\left[\begin{array}{c}
I_{a}^{S} \\
I_{b}^{S} \\
I_{c}^{S}
\end{array}\right]-\frac{j}{2}\left[\begin{array}{ccc}
G_{a} & G_{a b} & G_{a c} \\
G_{a b} & G_{b} & G_{b c} \\
G_{a c} & G_{b c} & G_{c}
\end{array}\right]\left[\begin{array}{c}
V_{a}^{S} \\
V_{b}^{S} \\
V_{c}^{S}
\end{array}\right] } \\
& {\left[\begin{array}{l}
\sum I_{a} \\
\sum I_{b} \\
\sum I_{c}
\end{array}\right]=\frac{j}{2}\left[\begin{array}{ccc}
B_{a} & B_{a b} & B_{a c} \\
B_{a b} & B_{b} & B_{b c} \\
B_{a c} & B_{b c} & B_{c}
\end{array}\right]\left[\begin{array}{l}
\sum V_{a} \\
\sum V_{b} \\
\sum V_{c}
\end{array}\right] }
\end{aligned}
$$

where, $\Delta V_{x}=V_{a}^{S}-V_{a}^{R}, \sum I_{x}=I_{x}^{S}+I_{x}^{R}, \sum V_{x}=V_{x}^{S}+V_{x}^{R}$ $x=a, b$, or $c$.

Further expanding Eqs. (11) and (12) yields the following:

$$
\begin{gathered}
\Delta V_{a}=Z_{a} I_{a}^{S}+Z_{a b} I_{b}^{S}+Z_{a c} I_{c}^{S}-\frac{j}{2}\left(G_{a} V_{a}^{S}+G_{a b} V_{b}^{S}+G_{a c} V_{c}^{S}\right) \\
\Delta V_{b}=Z_{a b} I_{a}^{S}+Z_{b} I_{b}^{S}+Z_{b c} I_{c}^{S}-\frac{j}{2}\left(G_{a b} V_{a}^{S}+G_{b} V_{b}^{S}+G_{b c} V_{c}^{S}\right) \\
\Delta V_{c}=Z_{a c} I_{a}^{S}+Z_{b c} I_{b}^{S}+Z_{c} I_{c}^{S}-\frac{j}{2}\left(G_{a c} V_{a}^{S}+G_{b c} V_{b}^{S}+G_{c} V_{c}^{S}\right) \\
\sum I_{a}=\frac{j}{2} \cdot\left[B_{a} \sum V_{a}+B_{a b} \sum V_{b}+B_{a c} \sum V_{c}\right]
\end{gathered}
$$

$$
\begin{aligned}
& \sum I_{b}=\frac{j}{2} \cdot\left[B_{a b} \sum V_{a}+B_{b} \sum V_{b}+B_{b c} \sum V_{c}\right] \\
& \sum I_{c}=\frac{j}{2} \cdot\left[B_{a c} \sum V_{a}+B_{b c} \sum V_{b}+B_{c} \sum V_{c}\right]
\end{aligned}
$$

In Eqs. (13)-(18), it is noticed that $G_{x}(x=a, b, c, a b$, $b c, a c)$ is a complex number, so that the following quantity is defined: $G x=S_{x}+j T_{x}$. In order to obtain an optimal estimate of the impedances, these six complex equations can be expanded into 12 real equations. Due to space considerations, these 12 equations are derived in the appendix. To simplify the expression, the following definitions are made.

Define $X$ to be the measurement vector, which is known and can be obtained from the PMU measurements.

$$
\begin{aligned}
& X=\left[x_{1}, x_{2}, \ldots, x_{24}\right]^{T} \\
& =\left[\operatorname{Re}\left(V_{a}^{S}\right), \operatorname{Im}\left(V_{a}^{S}\right), \operatorname{Re}\left(V_{b}^{S}\right), \ldots, \operatorname{Re}\left(V_{a}^{R}\right), \operatorname{Im}\left(V_{a}^{R}\right),\right. \\
& \left.\ldots, \operatorname{Re}\left(I_{a}^{S}\right), \operatorname{Im}\left(I_{a}^{S}\right), \ldots, \operatorname{Re}\left(I_{a}^{R}\right), \operatorname{Im}\left(I_{a}^{R}\right) \ldots\right]^{T}
\end{aligned}
$$

where, $\operatorname{Re}(\bullet)$ and $\operatorname{Im}(\bullet)$ yield the real and imaginary parts of the input arguments, respectively.

Define $\beta$ to be the unknown parameter vector, which is composed of the unknown impedance parameters.

$$
\begin{aligned}
& \beta=\left[\beta_{1}, \beta_{2}, \ldots, \beta_{30}\right]^{T}=\left[R_{a}, S_{a}, X_{a}, T_{a}, \ldots,\right. \\
& \left.R_{a b}, S_{a b}, X_{a b}, \ldots, T_{a c}, B_{a}, B_{b}, B_{c}, B_{a b}, B_{b c}, B_{a c}\right]^{T}
\end{aligned}
$$

With the definition given in Eq. (19), the following measurement vector is further defined as:

$$
\begin{aligned}
& Z=\left[x_{1}-x_{7}, x_{2}-x_{8}, x_{3}-x_{9}, x_{4}-x_{10},\right. \\
& x_{5}-x_{11}, x_{6}-x_{12}, x_{13}-x_{19}, x_{14}-x_{20}, x_{15}-x_{21}, \\
& \left.x_{16}-x_{22}, x_{17}-x_{23}, x_{18}-x_{24}\right]^{T}
\end{aligned}
$$

Based on the definitions above, these 12 equations are rearranged into matrix form as:

$$
Z=H \cdot \beta
$$

where, $H$ is a matrix formulated from the 12 equations referenced above and contains measurements while the measurement vector $Z$ contains PMU voltage and current measurements.

\subsection{Problem Formulation and Solution}

It has been demonstrated in Refs. $[1,4,6]$ that TL 
parameter identification is very sensitivity to the noise/error in the PMU measurements. For example, as shown in Ref. [1], a $1 \%$ error in the voltage measurements can lead to over $20 \%$ error in the calculated series resistance and reactance. In this project, even negative series resistance has been observed many times.

One important thing identified during the development of TPIS was that if physical constraints can be considered in the parameter identification process, accuracy of parameter estimation can be greatly improved. First, from Eq. (8), the following equality constraint can be identified as:

$$
\begin{gathered}
\beta_{2}=\beta_{1} \cdot \beta_{25}+\beta_{13} \cdot \beta_{28}+\beta_{21} \cdot \beta_{30} \\
\beta_{4}=\beta_{3} \cdot \beta_{25}+\beta_{15} \cdot \beta_{28}+\beta_{23} \cdot \beta_{30} \\
\beta_{14}=\beta_{1} \cdot \beta_{28}+\beta_{13} \cdot \beta_{26}+\beta_{21} \cdot \beta_{29} \\
\vdots \\
\beta_{10}=\beta_{21} \cdot \beta_{30}+\beta_{17} \cdot \beta_{29}+\beta_{9} \cdot \beta_{27} \\
\beta_{12}=\beta_{23} \cdot \beta_{30}+\beta_{19} \cdot \beta_{29}+\beta_{11} \cdot \beta_{27}
\end{gathered}
$$

As shown above, a total of 12 equality constraints can be added to the parameter estimation problem. To simplify the expression, these 12 equations can be collectively written as:

$$
f_{i}(\beta)=0 \quad \mathrm{i}=1,2, \ldots 12
$$

Second, although hand-calculated parameters only approximate the sag effect and neglect the temperature variation, it is believed that the true line parameters still stay within certain error band of the hand-calculated ones under different system operating conditions. The line parameters stored in the EMS database can be used as additional inequality constraints for the impedance parameters. Assuming the TL impedances stored in the EMS database have already been identified, it is reasonable to assume that the calculated impedance should follow the constraints below:

$$
\begin{gathered}
\left(1-\alpha_{R}\right) R_{a}^{E M S} \leq \beta_{1} \leq\left(1+\alpha_{R}\right) \cdot R_{a}^{E M S} \\
\left(1-\alpha_{X}\right) \cdot X_{a}^{E M S} \leq \beta_{2} \leq\left(1+\alpha_{X}\right) \cdot X_{a}^{E M S} \\
\vdots \\
\left(1-\alpha_{B c}\right) \cdot B_{c}^{E M S} \leq \beta_{27} \leq\left(1+\alpha_{B c}\right) \cdot B_{c}^{E M S}
\end{gathered}
$$

where, $\alpha_{R}, \alpha_{X}$, and $\alpha_{B c}$ are constants that define the error bands of the TL impedance parameters. It should be noted that it is not necessary to include all the above inequality constraints since some impedance parameters may not be immediately available from the EMS database. Generally speaking, the more the constraints are included, the better the results will be. To simplify the expression, Eqs. (29)-(31) can be collectively written as:

$$
l b_{j} \leq \beta_{j} \leq u b_{j} j=1,2,3 \ldots
$$

where, $l b_{j}$ and $u b_{j}$ represent the lower and upper boundaries identified.

Third, resistances are usually much smaller as compared to reactance for a transmission line:

$$
\begin{gathered}
\beta_{1} \leq \beta_{3} \\
\beta_{5} \leq \beta_{7} \\
\vdots \\
\beta_{21} \leq \beta_{23}
\end{gathered}
$$

A total of six additional constraints can be set up. Again, to simplify the expression, these equations are collectively written as:

$$
g_{k}(\beta) \leq 0 \quad k=1,2, \ldots 6
$$

Therefore, the TL parameter identification problem can be formulated as a least-squares curve-fitting problem subject to both equality and inequality constraints, as shown below:

$$
\begin{gathered}
\min _{\beta} \frac{1}{2} \cdot\|H \cdot \beta-Z\|_{2}^{2} \\
f_{i}(\beta)=0_{i}=1,2, \ldots 12
\end{gathered}
$$




$$
\begin{gathered}
g_{k}(\beta) \leq 0_{k=1}, 2, \ldots 6 \\
l b_{j} \leq \beta_{j} \leq u b_{j} j=1,2,3
\end{gathered}
$$

where, $\|\bullet\|_{2}^{2}$ denotes the square of the $L_{2}$-norm of the corresponding vector.

Once $\beta$ is calculated, the sequence impedances of the transmission line can be calculated using Eqs. (6) and (7).

\subsection{Bad Data Detection}

The classical method described in Refs. [10, 11] is used for bad data detection after solving the constrained least-squares. Bad data identification is achieved by checking the normalized residuals of each measurement, which proceeds as follows:

Step (1): solve the curve-fitting problem described in Eq. (32) and obtain the residual for each measurement point:

$$
r^{i}=z^{i}-H^{i} \cdot \beta, \quad i=1,2, \ldots, N
$$

Step (2): compute the normalized residual as:

$$
\left(r^{i}\right)^{\text {norm }}=\frac{r_{i}}{\sqrt{\Omega_{i i}}}, \quad i=1,2, \ldots, N
$$

where $\Omega_{i i}$ is the diagonal element of the matrix $\Omega$

$$
\Omega=H\left(H^{T} H\right)^{-1} H^{T}
$$

Step (3): find the largest normalized residual $r_{\max }^{\text {Norm }}$ and check whether it is larger than a predefined identification threshold $c$, for example 3.0:

$$
r_{\max }^{\text {Norm }}>c
$$

Step (4): if Eq. () does not hold, then no bad data will be suspected; otherwise, the data sample corresponding to the largest normalized residual is the bad data and should be removed from the data set.

Step (5): if bad data are detected and removed from the data set, the algorithm flow must return to step (1) and the process above must be repeated. Otherwise, this process ends and solutions are found.

\section{Online Implementation and Credibility Metric}

It is generally very difficult to determine the credibility of the calculated TL parameters, mainly because in reality, their corresponding true values are unknown and there is no way to make comparison. Most of the validation in existing research is based simulation in which the true values are assumed, a priori, to be known values and used throughout the simulation. Having a metric that can quantify the credibility of the calculated parameters is of critical importance in practice for applications like such and so.

In TPIS, for every five seconds, one set of impedance parameters is generated for the TL under consideration. PMU data collected during the five seconds are used to conduct bootstrapping. Bootstrapping is one type of re-sampling techniques that can be used to estimate the properties of an estimator by sampling from an approximating distribution. By resampling with replacement, it is assumed that each sample of PMU measurements is independent and identically distributed. The problem described by Eq. (37) is solved for each set of sampled PMU data to get one set of TL impedance parameters. Conduct the sampling many times so that the variances of the calculated parameters can be evaluated. Ratio of the variance of the parameters to the corresponding value stored in EMS database is used as the credibility metric for the parameter identification. That is, the parameter obtained is credible if the corresponding metric is smaller than a pre-determined threshold:

$$
\sigma(x) \leq \xi_{x} \quad x=R, X, \text { or } B_{c}
$$

where, $\sigma(x)$ stands for the variance of variable $x$.

During the implementation, two parameters are identified to be critical, as discussed below:

(1) Number of bootstrapping samples: this number defines how many times the program will acquire bootstrap samples from the set of available data points. 
It is strongly recommended that this number be larger than 30. As the number of bootstrap samples increases, the variances associated with the parameters decrease; however, execution time increases as the number of bootstrap samples increases and selecting arbitrarily large numbers may not be desirable. Tradeoff is needed;

(2) Number of data points in each bootstrap sample: this number determines how many data points are selected with each bootstrap sample. Increasing this number may lead to greater variance in the estimated parameters but will also increase the computation time. Tradeoff is needed.

Fig. 2 serves as an example to show the output of the TPIS during one 5-second interval. And Fig. 3 shows the flowchart for the online implementation of TPIS.

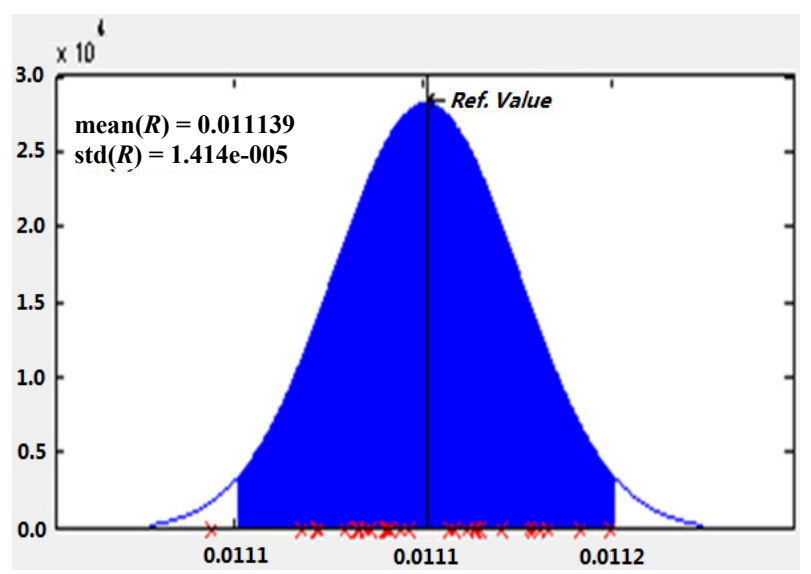

(a) Statistics for the calculated series resistance

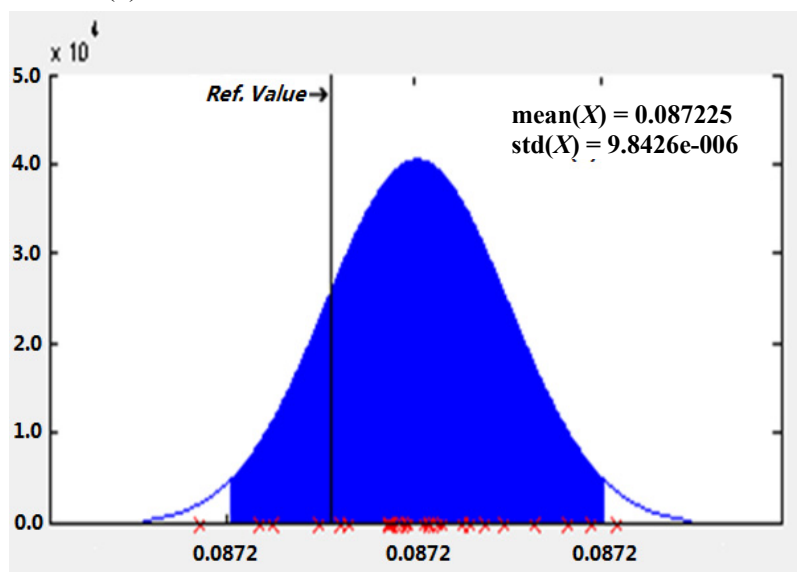

(b) Statistics for the calculated series reactance

Fig. 2 Credibility metric for the calculated impedance parameters.

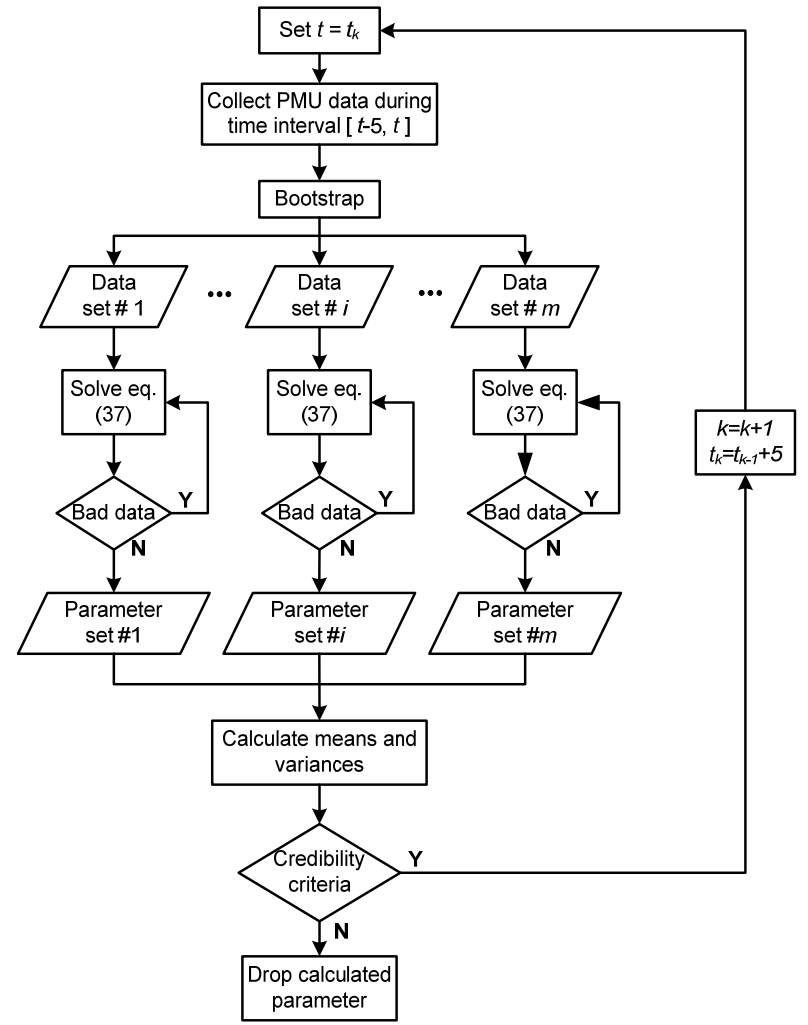

Fig. 3 Online implementation of TPIS.

\section{Numerical Example}

The TPIS has been implemented to a $525 \mathrm{kV}$ transmission line at CSG. This line is named as "Lai Bin-Wu Zhou \#2", which is the second transmission line connecting two cities (Lai Bin and $\mathrm{Wu}$ Zhou) in southern China. During a one-hour period, experiments were conducted and the calculated parameters for the line are shown below in Fig. 4-Fig. 6.

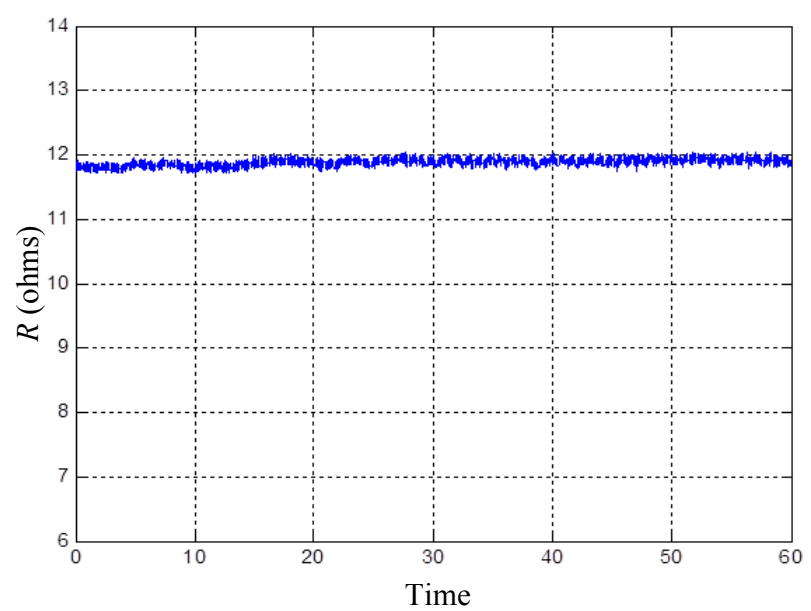

Fig. 4 Calculated series resistance. 
As can be seen from the plots, the impedance parameters do not vary much during the experiment period. In addition, as shown in Table 1, the calculated series impedance differs from the EMS database by about $7.8 \%$ while the shunt susceptance differs from the database by $7.7 \%$. These differences could be due to the inaccurate estimate of the line length as well as the specific loading conditions, and other ambience conditions such as temperature, wind speed at the moment when this experiment was conducted.

\section{Conclusion}

PMU has the potential to improve the accuracy of transmission line impedances. More accurate parameter means better power system modeling, faster and more accurate fault location as well as more economic system operation. China Southern Power

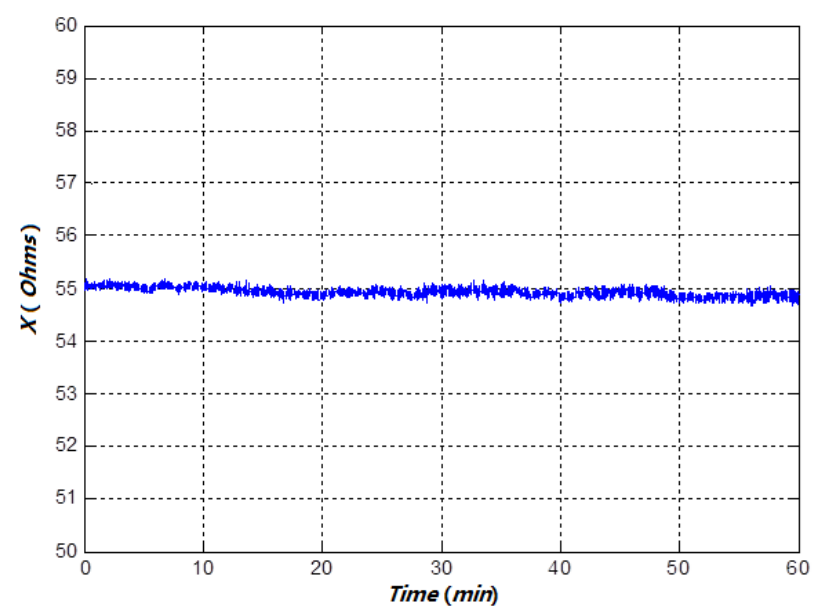

Fig. 5 Calculated series reactance.

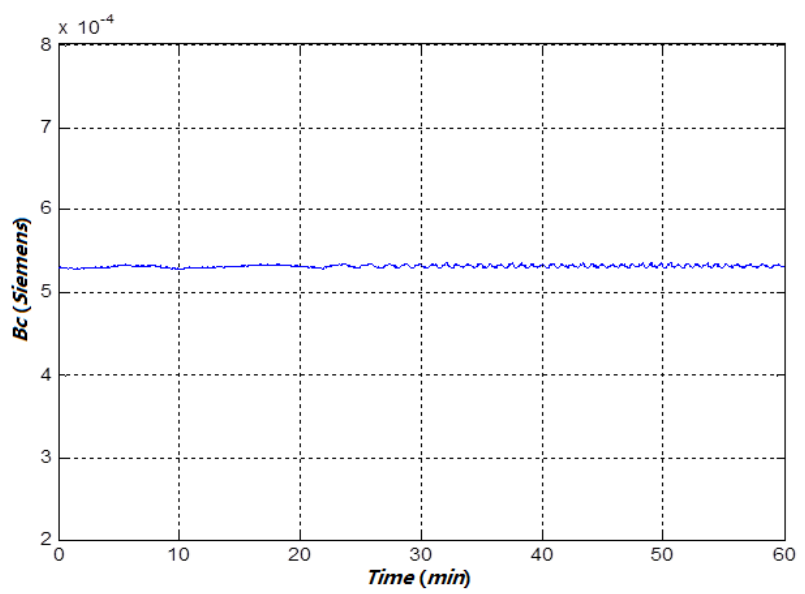

Fig. 6 Calculated shunt susceptance.
Table 1 Comparison between calculation and EMS database.

\begin{tabular}{llll}
\hline Quantity & $\begin{array}{l}\text { EMS } \\
\text { database }\end{array}$ & Calculated & $\begin{array}{l}\text { Difference } \\
(\%)\end{array}$ \\
\hline Series impedance $|Z|(\Omega)$ & 23.8 & 25.8 & 7.8 \\
Shunt susceptance $B c(\mathrm{~S})$ & $3.6 \mathrm{E}-04$ & $3.9 \mathrm{E}-04$ & 7.7 \\
\hline
\end{tabular}

Grid has developed an online TL parameter identification system. It was found that if the physical constraints associated with the TL were included, accuracy of the calculation can be greatly improved. Using bootstrapping, multiple sets of PMU measurements can be obtained so as to identify the credibility of the calculated parameters based on a proposed metric. A novel method is presented to calculate the TL sequence impedances.

\section{References}

[1] Shi, D., Tylavsky, D. J., Logic, N., and Koellner, K. M. 2008. "Identification of Short Transmission-Line Parameters from Synchrophasor Measurements." In Proceedings of the 40th North America Power Symposium, 1-8.

[2] Wilson, R. E., Zevenbergen, G. A., Mah, D. L., and Murphy, A. J. 1999. "Calculation of Transmission Line Parameters from Synchronized Measurements." Electric Machines \& Power Systems 27 (12): 1269-78.

[3] Liao, Y., and Kezunovic, M. 2007. "Optimal Estimate of Transmission Line Fault Location Considering Measurement Errors." IEEE Transactions on Power Delivery 22 (3): 1335-41.

[4] Shi, D., Tylavsky, D. J., Koellner, K. M., Logic, N., and Wheeler, D. E. 2011. "Transmission Line Parameter Identification Using PMU Measurements.” European Transactions on Electrical Power 21 (4): 1574-88.

[5] Janecek, E., Hering, P., Janecek, P., and Popelka, A. 2011. "Transmission Line Identification Using PMUs." In Proceedings of the 10th International Conference on EEEIC (Environment and Electrical Engineering), 1-4.

[6] Shi, D. 2009. "Utilising Synchrophasor Technology to Determine Transmission Line Impedance Parameters." Thesis, Arizona State University.

[7] Shi, D., Tylavsky, D. J., and Logic, N. 2012. "An Adaptive Method for Detection and Correction of Errors in PMU Measurements." IEEE Transactions on Smart Grid 3 (4): 1575-83.

[8] Zhang, Q., Vittal, V., Heydt, G. T., and Logic, N. 2011. "The Integrated Calibration of Synchronized Phasor 
Measurement Data in Power Transmission Systems." IEEE Transactions on Power Delivery 26 (4): 2573-81.

[9] Zhou, M., Centeno, V., Thorp, J. S., and Phadke, A. G. 2012. "Calibrating Instrument Transformers with Phasor Measurements." Electric Power Components and Systems 40 (14): 1605-20.
[10] Montgomery, D. C., Peck, E. A., and Vining, G. G. 2012 Introduction to Linear Regression Analysis. vol. 821. Hoboken, NJ: John Wiley \& Sons.

[11] Abur, A., and Exposito, A. G. 2004. Power System State Estimation: Theory and Implementation. Boca Raton. FL: CRC Press.

\section{Appendix}

Eqs. (13)-(18) are all complex equations and can be broken up into 12 real equations as shown below:

$$
\begin{aligned}
& \operatorname{Re}\left(\Delta V_{a}\right)=\operatorname{Re}\left(Z_{a}\right) \operatorname{Re}\left(I_{a}^{S}\right)-\operatorname{Im}\left(Z_{a}\right) \operatorname{Im}\left(I_{a}^{S}\right)+\operatorname{Re}\left(Z_{a b}\right) \operatorname{Re}\left(I_{b}^{S}\right) \\
& -\operatorname{Im}\left(Z_{a b}\right) \operatorname{Im}\left(I_{b}^{S}\right)+\operatorname{Re}\left(Z_{a c}\right) \operatorname{Re}\left(I_{c}^{S}\right)-\operatorname{Im}\left(Z_{a c}\right) \operatorname{Im}\left(I_{c}^{S}\right) \\
& +0.5 \cdot\left[G_{a} \operatorname{Im}\left(V_{a}^{S}\right)+G_{a b} \operatorname{Im}\left(V_{b}^{S}\right)+G_{a c} \operatorname{Im}\left(V_{c}^{S}\right)\right] \\
& \operatorname{Im}\left(\Delta V_{a}\right)=\operatorname{Re}\left(Z_{a}\right) \operatorname{Im}\left(I_{a}^{S}\right)+\operatorname{Im}\left(Z_{a}\right) \operatorname{Re}\left(I_{a}^{S}\right)+\operatorname{Re}\left(Z_{a b}\right) \operatorname{Im}\left(I_{b}^{S}\right) \\
& +\operatorname{Im}\left(Z_{a b}\right) \operatorname{Re}\left(I_{b}^{S}\right)+\operatorname{Re}\left(Z_{a c}\right) \operatorname{Im}\left(I_{c}^{S}\right)+\operatorname{Im}\left(Z_{a c}\right) \operatorname{Re}\left(I_{c}^{S}\right) \\
& -0.5 \cdot\left[G_{a} \operatorname{Re}\left(V_{a}^{S}\right)+G_{a b} \operatorname{Re}\left(V_{b}^{S}\right)+G_{a c} \operatorname{Re}\left(V_{c}^{S}\right)\right] \\
& \operatorname{Re}\left(\Delta V_{b}\right)=\operatorname{Re}\left(Z_{a b}\right) \operatorname{Re}\left(I_{a}^{S}\right)-\operatorname{Im}\left(Z_{a b}\right) \operatorname{Im}\left(I_{a}^{S}\right)+\operatorname{Re}\left(Z_{b}\right) \operatorname{Re}\left(I_{b}^{S}\right) \\
& -\operatorname{Im}\left(Z_{b}\right) \operatorname{Im}\left(I_{b}^{S}\right)+\operatorname{Re}\left(Z_{b c}\right) \operatorname{Re}\left(I_{c}^{S}\right)-\operatorname{Im}\left(Z_{b c}\right) \operatorname{Im}\left(I_{c}^{S}\right) \\
& +0.5 \cdot\left[G_{a b} \operatorname{Im}\left(V_{a}^{S}\right)+G_{b} \operatorname{Im}\left(V_{b}^{S}\right)+G_{b c} \operatorname{Im}\left(V_{c}^{S}\right)\right] \\
& \operatorname{Im}\left(\Delta V_{b}\right)=\operatorname{Re}\left(Z_{a b}\right) \operatorname{Im}\left(I_{a}^{S}\right)+\operatorname{Im}\left(Z_{a b}\right) \operatorname{Re}\left(I_{a}^{S}\right)+\operatorname{Re}\left(Z_{b}\right) \operatorname{Im}\left(I_{b}^{S}\right) \\
& +\operatorname{Im}\left(Z_{b}\right) \operatorname{Re}\left(I_{b}^{S}\right)+\operatorname{Re}\left(Z_{b c}\right) \operatorname{Im}\left(I_{c}^{S}\right)+\operatorname{Im}\left(Z_{b c}\right) \operatorname{Re}\left(I_{c}^{S}\right) \\
& -0.5 \cdot\left[G_{a b} \operatorname{Re}\left(V_{a}^{S}\right)+G_{b} \operatorname{Re}\left(V_{b}^{S}\right)+G_{b c} \operatorname{Re}\left(V_{c}^{S}\right)\right] \\
& \operatorname{Re}\left(\Delta V_{c}\right)=\operatorname{Re}\left(Z_{a c}\right) \operatorname{Re}\left(I_{a}^{S}\right)-\operatorname{Im}\left(Z_{a c}\right) \operatorname{Im}\left(I_{a}^{S}\right)+\operatorname{Re}\left(Z_{b c}\right) \operatorname{Re}\left(I_{b}^{S}\right) \\
& -\operatorname{Im}\left(Z_{b c}\right) \operatorname{Im}\left(I_{b}^{S}\right)+\operatorname{Re}\left(Z_{c}\right) \operatorname{Re}\left(I_{c}^{S}\right)-\operatorname{Im}\left(Z_{c}\right) \operatorname{Im}\left(I_{c}^{S}\right) \\
& +0.5 \cdot\left[G_{a c} \operatorname{Im}\left(V_{a}^{S}\right)+G_{b c} \operatorname{Im}\left(V_{b}^{S}\right)+G_{c} \operatorname{Im}\left(V_{c}^{S}\right)\right] \\
& \operatorname{Im}\left(\Delta V_{c}\right)=\operatorname{Re}\left(Z_{a c}\right) \operatorname{Im}\left(I_{a}^{S}\right)+\operatorname{Im}\left(Z_{a c}\right) \operatorname{Re}\left(I_{a}^{S}\right)+\operatorname{Re}\left(Z_{b c}\right) \operatorname{Im}\left(I_{b}^{S}\right) \\
& +\operatorname{Im}\left(Z_{b c}\right) \operatorname{Re}\left(I_{b}^{S}\right)+\operatorname{Re}\left(Z_{c}\right) \operatorname{Im}\left(I_{c}^{S}\right)+\operatorname{Im}\left(Z_{c}\right) \operatorname{Re}\left(I_{c}^{S}\right) \\
& -0.5 \cdot\left[G_{a c} \operatorname{Re}\left(V_{a}^{S}\right)+G_{b c} \operatorname{Re}\left(V_{b}^{S}\right)+G_{c} \operatorname{Re}\left(V_{c}^{S}\right)\right] \\
& \operatorname{Re}\left(\sum I_{a}\right)=-0.5 \cdot\left[B_{a} \operatorname{Im}\left(\sum V_{a}\right)+B_{a b} \operatorname{Im}\left(\sum V_{b}\right)+B_{a c} \operatorname{Im}\left(\sum V_{c}\right)\right] \\
& \operatorname{Im}\left(\sum I_{a}\right)=0.5 \cdot\left[B_{a} \operatorname{Re}\left(\sum V_{a}\right)+B_{a b} \operatorname{Re}\left(\sum V_{b}\right)+B_{a c} \operatorname{Re}\left(\sum V_{c}\right)\right] \\
& \operatorname{Re}\left(\sum I_{b}\right)=-0.5 \cdot\left[B_{a b} \operatorname{Im}\left(\sum V_{a}\right)+B_{b} \operatorname{Im}\left(\sum V_{b}\right)+B_{b c} \operatorname{Im}\left(\sum V_{c}\right)\right] \\
& \operatorname{Im}\left(\sum I_{b}\right)=0.5 \cdot\left[B_{a b} \operatorname{Re}\left(\sum V_{a}\right)+B_{b} \operatorname{Re}\left(\sum V_{b}\right)+B_{b c} \operatorname{Re}\left(\sum V_{c}\right)\right] \\
& \operatorname{Re}\left(\sum I_{c}\right)=-0.5 \cdot\left[B_{a c} \operatorname{Im}\left(\sum V_{a}\right)+B_{b c} \operatorname{Im}\left(\sum V_{b}\right)+B_{c} \operatorname{Im}\left(\sum V_{c}\right)\right] \\
& \operatorname{Im}\left(\sum I_{c}\right)=0.5 \cdot\left[B_{a c} \operatorname{Re}\left(\sum V_{a}\right)+B_{b c} \operatorname{Re}\left(\sum V_{b}\right)+B_{c} \operatorname{Re}\left(\sum V_{c}\right)\right]
\end{aligned}
$$

